\title{
Entwicklungen und Innovationen beim Übertragungsnetzbetreiber
}

\author{
G. Achleitner OVE, K. Reich OVE, H. Popelka OVE \\ Online publiziert am 22. Jänner 2019 \\ (c) Springer-Verlag GmbH Austria, ein Teil von Springer Nature 2019
}

Die Energiewende und die Netzintegration der Erneuerbaren sowie netzbetriebliche und marktseitige Entwicklungen stellen APG vor neue Herausforderungen. APG begegnet diesen mit innovativen Ansätzen, neuen Technologien und zahlreichen Forschungsprojekten für den Netzbetrieb und bei den Netzanlagen. Im Rahmen des Vortrages bei der heurigen OGE-Fachtagung werden dazu Beispiele aus dem Bereich der Sekundärtechnik bei Umspannwerken und bei Hochspannungsleitungen gebracht.

Die Energiewende erfordert nicht nur im Netzbetrieb neue Ansätze, sondern auch in der Errichtung und im Betrieb von Umspannwerken. Im Bereich der Sekundärtechnik der Umspannwerke wurden in den letzten Jahren mehrere innovative Ideen und Ansätze bei und mit APG initiiert, wovon zwei besonders zu erwähnen sind. Diese wurden in Zusammenarbeit mit der TU Graz und österreichischen Firmen, insbesondere Sprecher Automation, bis zur Praxisreife entwickelt.

Die erste Innovation betrifft die Erdschlussortung in 110-kVNetzen. Die Idee basiert auf bestehenden Distanzschutzgeräten, die nun zusätzlich für die Erdschlussortung verwendet werden. Der Vorteil des neuen Verfahrens ist, dass nunmehr Fehler nicht nur erkannt, sondern auch der Fehlerort bestimmt werden kann. An Hand von unzähligen Versuchen konnte die Genauigkeit des entwickelten Verfahrens gezeigt werden. Derzeit laufen erste Praxistests, in denen sich die Innovation bereits bewähren konnte.

Die zweite Innovation betrifft eine Batteriefehlerortung in den Eigenbedarfsanlagen für Umspannwerke. Derzeit sind Batteriefehler sehr schwer zu erkennen und zu lokalisieren. Dies geht oft mit Ausund Umschaltungen von Verbrauchern wie Leittechnik oder Schutzgeräten einher. Um die Eingrenzung zu vereinfachen und betriebliche Nachteile zu vermeiden, wurde ein Verfahren entwickelt, das sehr einfach funktioniert und auch sehr hochohmige Isolationsfehler erfassen kann. Dieses Verfahren wurde in einer Prototypenanlage ausführlich getestet, und die Batteriefehlerortung wird mittlerweile seit einem Jahr bereits standardmäßig in den APG-Umspannwerken eingebaut.

Auch wenn die Technik der Hochspannungsfreileitung seit über 120 Jahren angewandt und immer wieder weiterentwickelt wurde, sind aktuell Innovationen, die mehrfach auf Digitalisierung und modernen Datenverarbeitungs-Tools basieren, im Gange. In den letzten Jahren sind zahlreiche neue Anwendungen entstanden bzw. befinden sich derzeit in Entwicklung. Dabei sind drei Schwerpunkte zu erkennen:

Der erste Schwerpunkt liegt in einer verbesserten Betriebsüberwachung. Hier werden insbesondere Verbesserungen in der $(n-1)$ Sicherheit durch Überwachung der Übertragungskapazität (dynamic line rating) angestrebt, wobei neben Echtzeitdaten und -monitoring die Kapazitätsprognose mit unterschiedlichen Zeithorizonten für die Betriebsplanung eine große Rolle spielt. Verschiedene Sensortypen, die unterschiedliche Primärgrößen messen, und Prognosesysteme suchen derzeit eine Etablierung am Markt.
Weitere Innovationen betreffen das Monitoring von Umweltaspekten. Dies kann im Rahmen von Projekten für die Planung (IstZustands-Erfassung) sowie für die Dokumentation bzw. den Nachweis der Einhaltung von Auflagen nach der Projektrealisierung relevant sein. Ein Beispiel für ein solches Monitoring ist eine möglichst exakte Erfassung von Schallemissionen von Freileitungen (v. a. Corona-Geräusche). APG hat in diesem Bereich gemeinsam mit mehreren Industriepartnern und mit der TU Graz ein Forschungsprojekt initiiert, das einen neuartigen Schallsensor im unmittelbaren Nahebereich des Leiterseiles zur Anwendung bringt (d. h. der Sensor wird direkt auf den Leiterseilen der Hochspannungsleitung montiert). Der Einfluss von störenden Umgebungsgeräuschen kann so minimiert werden und die Auswertung wesentlich genauer erfolgen. Weitere Beispiele von Innovationen im Bereich der Umweltaspekte sind das Erkennen von Aneisungen oder Fremdeinwirkungen, wie Vögel im Nahebereich von Freileitungen.

Ein dritter Schwerpunkt ist die technische Anlagen- und Zustandsüberprüfung mit verbesserter Sensorik und teil-automatisierten Auswertungen mittels Bilderkennung (künstliche Intelligenz). Dabei kommen vermehrt Multikopter (Drohnen) und die Laser-ScanTechnologie zum Einsatz. Mit Hilfe dieser neuen Methoden werden künftig eine automatisierte Beurteilung des Abstandes zu Bewuchs (Trassenfreihaltung) und Festlegung von Rückschnittmaßnahmen sowie deren Kontrolle möglich. Ein weiterer Anwendungsbereich ist die Erkennung des Anlagenzustandes und die Ableitung von Instandhaltungsmaßnahmen.
Kurzfassung eines Vortrags bei der 56. Fachtagung der Österreichischen Gesellschaft für Energietechnik (OGE) im OVE, die am 18. und 19. Oktober 2018 in Wien stattfand.

Achleitner, Georg, Austrian Power Grid AG, Wagramer Straße 19, 1220 Wien, Österreich (E-Mail: Georg.achleitner@apg.at); Reich, Klemens, Austrian Power Grid AG, Wagramer Straße 19, 1220 Wien, Österreich (E-Mail: klemens.reich@apg.at); Popelka, Herbert, Austrian Power Grid AG, Wagramer Straße 19, 1220 Wien, Österreich (E-Mail: herbert.popelka@apg.at) 